\title{
La Investigación Básica. La Investigación en Ciencias Fisiológicas: Bioquímica, Biología Molecular y Fisiología. Cuestiones Previas
}

\author{
Basic Research. Research in Physiological Sciences: Biochemistry, \\ Biophysics, Molecular Biology and Physiology. Some prior considerations
}

Constancio González Martínez

Facultad de Medicina. Universidad de Valladolid. Valladolid

La conferencia impartida los últimos días de enero de 2004 en las Dependencias del Instituto Carlos III (Madrid) ante un grupo de colegas latinoamericanos, y que transcribo en el escrito que sigue, ha tenido como objetivo fundamental tratar de conjugar algunas ideas sobre investigación con la realidad socioeconómica de los países de Latinoamérica. En la primera parte de la conferencia he dado un concepto de Investigación y he enfatizado el valor que la consideración de la teleología de los fenómenos biológicos tiene en el avance de las propias ciencias biológicas.

A continuación, he pasado revista de forma muy rápida a las Normas del Bien Hacer en le Laboratorio o Good Laboratory Practices, y he presentado unos pocos datos objetivos sobre la realidad económica de los países latinoamericanos. En una segunda parte, he tratado de promover en nuestros colegas latinoamericanos una actitud reivindicativa que les ayude a pedir a sus gobernantes y a defender frente a sus conciudadanos, la necesidad de implementar una política científica clara como una prioridad nacional. Tal reivindicación estaría apoyada en el reconocimiento de que la investigación es una herramienta para aprovechar la capacidad intelectual de los ciudadanos de cada país, de que la investigación es un medio para aprovechar recursos de organismos internacionales, de que la investigación es una fuente de cultura que contribuye a la creación de una identidad nacional y, finalmente, de que la investigación es una actividad generadora de riqueza y bienestar. Concluyo la conferencia señalando que la investigación básica, y por tanto la investigación en Ciencias Fisiológicas, está

\footnotetext{
Correspondencia:

Constancio González Martínez

Facultad de Medicina. Universidad de Valladolid

C/ Ramón y Cajal s/n

Valladolid

E-mail: constanc@ibgm.uva.es
}

ensamblada tan estrechamente con la investigación aplicada que constituyen una realidad única.

Palabras clave: Investigación básica, normas del buen hacer en el laboratorio, producto interior bruto, burocracia, prioridades en investigación.

This article is a transcription of a conference addressed to a group of colleagues from Latin America at the end of January 2004, in the Instituto Carlos III (Madrid). Its main purpose was to conjugate some general ideas on scientific research with known facts on the socioeconomic reality of Latin American countries. In the initial part of the talk I put forward a definition of research, emphasizing the great value of considering the teleology of biological phenomena for the advance of biological sciences. A succinct consideration of the importance of Good Laboratory Practices, especially if there is not a tradition of research, drove my talk to the presentation of some basic data on the socioeconomic situation of the Latin American countries. In the second half of the conference my efforts were directed to incite our Latin American colleagues to demand from their politicians, and to justify in front of their fellow citizens, the necessity of implementing a program on scientific research as a national priority. Such demand should be justified on the basis of the recognition that research represents a way to correctly use the intellectual capital of the citizens of every country and a mean to profit from free international resources, that research is a source of culture, contributing to the national identity of any given country, and finally, on the fact that research is an activity that generates wealth. I concluded my talk pointing out that basic research, and therefore research in physiological sciences, is assembled so tight with basic research that they conform a unique reality.

Key words: Basic research, good laboratory practices, gross national product, bureaucracy, research priorities. 
Cuando recibí el encargo del Profesor Gual de hablar en este foro, me consideré afortunado porque mis experiencias con colegas de ultramar han sido siempre enriquecedoras y agradables. Se me pidió hablar de investigación básica en general, aunque luego se concretó que hablara de investigación en Ciencias Fisiológicas, que evidentemente es una parcela prototipo de la investigación básica. Sin embargo, en el contexto preciso en que estamos, principios del siglo XXI y la realidad socioeconómica de los países de Latinoamérica, creo que es de interés hacer algunas consideraciones previas.

Así, no me resisto a dar una definición operativa de lo que es la investigación y que muestro en el cuadro 1 . Destaco en esta definición que la búsque-

Cuadro 1. Concepto de Investigación

Investigar es buscar el qué o el cómo (investigación descriptiva), el para qué (investigación de la teleología o finalidad) y el por qué (investigación de la causalidad) de las estructuras, procesos o fenómenos.

La búsqueda del para qué es un atributo exclusivo, o casi exclusivo, de la investigación biológica, porque sólo las estructuras y procesos biológicos tienen la propiedad intrínseca de la finalidad

da del para qué, de la finalidad de las estructuras o de los procesos, es un atributo exclusivo de las estructuras o fenómenos biológicos. Así, por ejemplo, de una reacción química que tiene lugar en el tubo de ensayo no es pertinente preguntarse cuál es su objetivo intrínseco o cuál es la finalidad intrínseca de la reacción; sin embargo, respecto a la misma reacción química descrita en unas células, sí que es pertinente que nos preguntemos, ¿para qué ocurre esa reacción?, ¿cuál es su finalidad en la organización funcional global de las células?. Del mismo modo, no tiene sentido que nos preguntemos el para qué de la forma de los cristales de hielo o de los minerales, pero sí que lo tiene el que nos hagamos esa pregunta de la forma de las hojas de los árboles o de las distintas células que componen los seres vivos. Por tanto, este interrogante respecto a las estructuras o procesos vitales, sujeto de la investigación de las ciencias fisiológicas en general debe presidir la práctica totalidad de nuestras investigaciones, debe ser un valor añadido al quehacer del investigador en ciencias biológicas. Cannon ${ }^{1}$ en su libro autobiográfico "The Way of an Investigator" enfatiza el valor especial que tiene la consideración de la finalidad en los fenómenos biológicos observados con una cita de E. von Bruecke, un fisiólogo alemán, que dice textualmente: "Teleology is a lady without whom no biologist can live. Yet, he is ashamed to be shown himself with her in public". El valor añadido de preguntarnos el para qué de los fenómenos observados radica en que con gran frecuencia tal pregunta lleva a sugerirnos nuevos experimentos, nuevas rutas en nuestra actividad inquisitoria. Además, en el contexto específico de principios del siglo XXI, preguntarnos el para qué de las estructuras o procesos biológicos es importante por dos razones:

- Primera, porque la era que vivimos es propicia para olvidar el atributo de finalidad de todo lo biológico. Esto es así, porque la investigación actual en las ciencias biológicas es fundamentalmente analítica y muy especializada, lo que proporciona un conocimiento enormemente fragmentado de los procesos biológicos desde el que a veces resulta muy difícil percibir la finalidad intrínseca de aquello que se investiga.

- Segunda, porque la interdisciplinaridad de las ciencias y el propio mercado laboral hacen que gran número de investigadores en biología, especialmente entre los mas jóvenes, provenga de áreas no biológicas (química, física, ingenierías), y por tanto que carezcan de la idea de la finalidad que la formación como biólogos proporciona. Estos investigadores han de aprender a preguntarse el para qué de lo que observan, de otro modo pierden el valor añadido que se obtiene en la búsqueda de la finalidad intrínseca de lo observado.

Otro aspecto formal, al que quiero referirme es al Método Científico. Desde que Claude Bernard publicara en 1865 la Introducción al Estudio de la Medicina Experimental, son muchos los autores que han escrito sobre el tema, pero al entender de Peter Brian Medawar, Premio Nóbel de Medicina en 1960, no ha habido nadie que haya escrito mejor y más inteligentemente que el propio Claude Bernard. Por ello me atrevería a aconsejar a todos aquellos que tenga-

1. CANNON, W. B. The way of an investigator. New York: Hafner Publishing Co., 1968. 
mos tareas de formar a futuros científicos, y esto incluye a todos los profesores universitarios, que recomendemos encarecidamente la lectura de la Introducción al Estudio de la Medicina Experimental. No obstante, quiero llamar la atención sobre el hecho de que la lectura, y aún el dominio, de los conceptos vertidos por Claude Bernard no es garantía, según opiniones de Cajal y de Medawar, a la hora de hacer descubrimientos científicos (Cuadro 2).

\section{Cuadro 2. Valor limitado del Método Científico}

“... He leído varios libros sobre los aspectos formales de la ciencia, sobre la filosofía de la ciencia y sobre el método científico, y todos ellos son excelentes para hacer pensar, pero de ningún modo son eficaces para enseñar a descubrir o para crear investigadores".

Santiago Ramón y Cajal. Reglas y Consejos sobre la investigación Científica. Los Tónicos de la Voluntad (1897).

... Ask a scientist what he conceives the scientific method to be, and he will adopt an expression that is at once solemn and shifty-eyed: solemn, because he feels he ought to declare an opinion; shiftyeyed, because he is wondering how to conceal the fact that he has no opinion to declare.

Sir Peter Brian Medawar. Induction and Intuition in Scientific Thought (1969).

Así pues, si la formación humanística y la adquisición de una concepción adecuada de lo que son los seres vivos aconsejan encarecidamente la lectura de los aspectos formales del Método Científico, en particular de la obra de Claude Bernard, el éxito de nuestras investigaciones no va a venir de ahí. En el transcurso de las jornadas y en la discusión de mi presentación, estuvimos de acuerdo en que a hacer ciencia se aprende haciéndola. Resulta a veces difícil trazar las etapas del método científico en una aportación (o descubrimiento) científica: la observación, la hipótesis y el diseño experimental constituyen con mucha frecuencia un momento único. La ejecución del experimento, su análisis y la rectificación de la hipótesis original o la formulación de una nueva hipótesis con un diseño nuevo suelen representar también otro momento único. Sólo cuando un investigador hace un análisis sereno y minucioso de su quehacer es capaz de describir las etapas formales del método científico.

También dentro del marco conceptual o formal, hay otras cuestiones previas, que sí que pueden ayudar a crear ciencia, que sí que pueden contribuir al éxito de nuestras investigaciones. Me refiero a las "Good Laboratory Practices" (GLP; Normas del buen hacer en el laboratorio; cuadro 3).

Cuadro 3. Concepto y objetivo de las GLP

Concepto: Good Laboratory Practice is defined in the OECD Principles as: "... a quality system concerned with the organisational process and the conditions under which non-clinical health and environmental safety studies are planned, performed, monitored, recorded, archived and reported."

Objetivo: The purpose of these Principles of Good Laboratory Practice is thus to promote the development of quality test data and to provide a managerial tool to ensure a sound approach to the management, including conduct, reporting and archiving of laboratory studies.

HANDBOOK. GOOD LABORATORY PRACTICE (GLP). Quality practices for regulated non-clinical research and development. UNDP/World Bank/wHO. 2001

El concepto de las GLP se comprende mejor si se tiene en cuenta el origen de las propias normas, que están recogidas con pequeñas variantes en documentos de múltiples organizaciones supranacionales. El concepto de GLP surge en los Estados Unidos frente a la duda de la validez de los datos preclínicos o de las investigaciones básicas enviadas a la FDA (Federal Drug Administration) para obtener la licencia de ensayos clínicos de nuevos fármacos. Las inspecciones de los estudios y de los laboratorios demostraron una serie de deficiencias relativamente frecuentes tales como que: los datos controles aportados eran datos obtenidos de animales controles de estudios previos, pero no del estudio concreto bajo auditoría; los datos finales aportados se basaban en el análisis de un número a veces reducido y muy dispar en el tamaño de las muestras de animales; las necropsias de los animales incluidos en los estudios no eran sistemáticas, no abarcaban a todos los animales del estudio; había carencias o faltas de registro en los cuadernos de protocolos del laboratorio, de tal forma que afirmaciones que se hacían en el informe final no constaban en los cuadernos de laboratorio; etc. El Senador E. Kennedy en el Congreso de los EE.UU. hizo públicas estas deficiencias y promovió que la FDA publicará las primeras regulaciones o normas del bien hacer en laboratorio o GLP en 1976 en relación con la investigación preclínica. A instancias de la EPA 
(Environmental Protection Agency) muy pronto aparecieron regulaciones similares en el área de las investigaciones químicas y de pesticidas.

En el ámbito internacional la Organization for Economical Cooperation and Development (OECD) promovió la elaboración unos Principios generales de GLP para todos los países miembros con el objetivo de evitar duplicación de esfuerzos y experimentos: los experimentos hechos en un país siguiendo las GLP de la OCDE serían válidos en todos los países miembros (cuadro 4).

\section{Cuadro 4. Parcelas generales a las que hacen referencia} las GLP.

- Resources: Organization, personnel, facilities, equipment.

- Rules: Protocols, Standard Operating Procedures, concept of the Study Director as the pivotal point of study control

- Characterization: test items, test systems.

- Documentation: Raw data, final report, archives.

- Quality assurance: Independence from study conduct

Correspondería a cada país miembro de la OECD la creación de un sistema de control, vigilancia e inspección sobre el cumplimiento en todos sus términos de las GLP emanadas de los acuerdos internacionales. Los países en los que no existan las oficinas de control deben ser instancias internacionales (inspectores de la FDA o de la Comunidad Europea) las que vigile y sancione el cumplimiento de las GLP. De forma muy esquemática, y sin entrar en subdivisiones ni en detalles paso a describir de manera sucinta las grandes parcelas del quehacer científico en la que inciden las GLP:

\section{Recursos (Resources):}

a) Personal. El laboratorio debe poseer un organigrama del personal que trabaja en él, con una descripción de los puestos de trabajo y con las tareas específicas de cada puesto. Debe haber una definición inequívoca del director del estudio, ya que él es la clave en el control de calidad del estudio; durante el proceso del mismo es responsable de las correcciones que en el estudio hayan de introducirse.

b) Facilidades y equipo. Los laboratorios han de tener dimensiones apropiadas para el personal y para el equipo que han de acoger. El hacinamiento va a generar conflictos personales, contaminaciones innecesarias, etc. Han de existir facilidades de agua, electricidad y ambientación eficaz, animalarios con salas de cuarentena y, llegado el caso, con salas estériles, etc. Se cuidarán los equipos y sus calibraciones para que los datos sean útiles. Se diseñaran programas de verificación periódica de los equipos etc., etc. Muchos de estos aspectos son obvios y se realizan de forma rutinaria en los laboratorios con tradición, pero hay que implementarlos de forma específica cuando se ponen en marcha laboratorios nuevos.

\section{Reglas (Rules):}

a) Protocolos. Las etapas del experimento a realizar han de describirse en el protocolo que quedará recogido en el cuaderno de protocolos. Corresponde al director su verificación y debe firmarlo cada día o cada serie de experimentos del mismo tipo. El cuaderno ha de poseer hojas numeradas e irremplazables. Las modificaciones al protocolo ha de realizarlas, o en su caso visarlas, el director del estudio, etc.

b) Procedimientos específicos del experimento. El protocolo no puede recoger todos los incidentes del experimento ni las modificaciones impuestas por el propio desarrollo del experimento. Estos detalles han de estar reflejados en el cuaderno de laboratorio para poder reconstruir cada uno de los experimentos concretos. El cuaderno de protocolos pertenece al laboratorio y debe estar en el laboratorio.

c) El director del estudio. Es el principal responsable del control de la calidad del estudio y debe aceptar por escrito esa responsabilidad.

\section{Caracterización (characterization):}

Hace referencia al conocimiento que a priori ha de tenerse de los fármacos o drogas a ensayar en los experimentos, de su toxicidad; al conocimiento de las especies animales en las que se van realizar los experimentos (cepas, estado de salud, edad, valores de constantes biológicas, etc.); al conocimiento de los equipos de medida, etc.

\section{Documentación (Documentation):}

a) Datos crudos: debe haber un registro claro y numerado de los datos crudos tal y como se obtienen en cada experimento, con los comentarios pertinentes que deben ser en gran parte copia de los datos del cuaderno de laboratorio y de otras circunstancias acaecidas durante los propios procedimientos analíticos, etc.

b) El informe del estudio. Su redacción compete al director del estudio. Debe ser preciso al relatar los hallazgos y deslindar muy bien los hechos de las interpretaciones. La publicación que pueda surgir del estudio, ha de tener las mismas virtudes que ha tenido el estudio.

c) Archivo. Es derecho y deber del laboratorio, y 
el director ha de implementarlo y ha de asegurar que hay un archivo correcto de todos los estudios, para si fuera necesario poder repetirlos. El archivo del laboratorio consta de los cuadernos de protocolos, archivos de datos, análisis estadísticos, publicaciones e informes, etc. etc.

\section{Control de calidad (Quality assurance):}

Ha de ser ejercido en forma de auditorias, con auditores que no sean componentes del equipo ejecutor del estudio, que sean independientes. Es evidente que el director del estudio debe formar parte del equipo auditor y servir como guía y proveedor de la información y documentación que los auditores externos soliciten.

Estos aspectos referidos a las GLP tienen gran trascendencia para la investigación en los países en desarrollo. Por ejemplo, la WHO (World Health Organization) en su programa de investigación en enfermedades tropicales, que idealmente debe realizarse en los países más afectados, entre los que los países latinoamericanos forman un grupo cualificado, sólo puede financiar las investigaciones desde el descubrimiento de una droga hasta su envasado y comercialización (ver Diagrama 1), si los laborato-

Diagrama 1. Proceso global desde el descubrimiento de un fármaco a su comercialización.

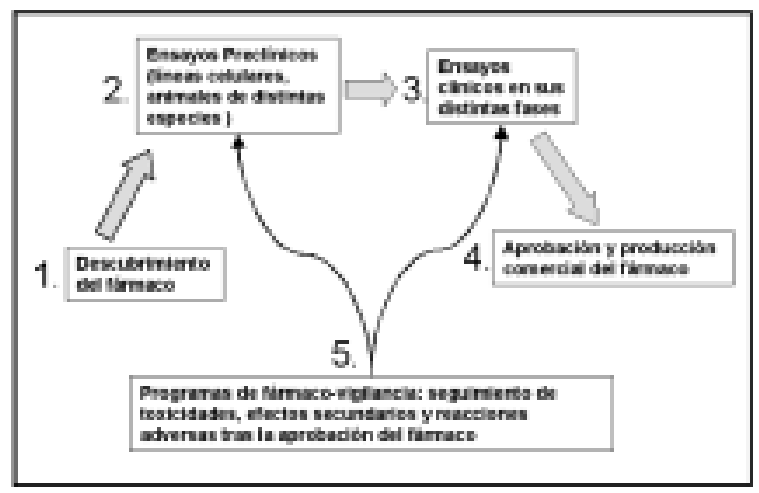

rios de los países involucrados cumplen con los Principios de GLP internacionales. Ello implica que las legislaciones nacionales tienen que recoger las normas de GLP, y hacerlas cumplir en todas las etapas de la investigación. En el ejemplo del diagrama se trataría del proceso global desde el descubrimiento hasta la comercialización de un medicamento, e incluiría las GLP, las good clinical practices (GCP) y las good manufacturing practices (GMP).

En resumen ha de promoverse en los países en desarrollo la introducción de GLP en todas las etapas de la investigación, de otro modo estos países se verán privados, no ya sólo de los fondos de organizaciones internacionales públicas como la WHO, sino de fondos de las grandes compañías farmacéuticas que no van a hacer el intento siquiera de contratar sus servicios; tampoco los propios países van a poder hacer el ofrecimiento de realizar el desarrollo de las medicaciones. Hay que percatarse de que las GLP, además del buen hacer de cada día de los científicos, implican políticas científicas implementadas desde los gobiernos, de forma especial en lo que se refiere a los recursos. Los gobiernos deben asegurar la disponibilidad de laboratorios, equipos materiales y humanos e infraestructuras adecuadas (agua corriente, electricidad, animalarios, etc.) Además las legislaciones estatales han de incidir sobre el resto de las facetas o parcelas a las que hace referencia ese listado resumido de GLP. Los gobernantes han de darse cuenta de que la carencia de instalaciones con las características enunciadas en los GLP imposibilita el acceso a recursos públicos o privados externos al propio país, que, a su vez, podrían ser fuente de plusvalías para programas de investigación adicionales.

Haciendo un quiebro en el discurso que estoy haciendo, quiero redirigirme a la investigación en Ciencias Fisiológicas, para finalizar volviendo a estos aspectos formales. La primera pregunta que debemos formularnos es si en los países en desarrollo hay que hacer investigación, y la segunda si hay que hacer investigación básica ${ }^{2}$, tal y como es la investi-

2. Me he referido con anterioridad a la investigación básica en contraposición tácita con la investigación aplicada. Por ello, y aún cuando soy escéptico respecto a las diferencias entre investigaciones básicas y aplicadas, quiero, siguiendo una tradición muy al uso acotar estos y otros conceptos en esta nota.

-Investigación básica: conjunto de actividades humanas que están encaminadas y consiguen ampliar el conocimiento sobre nosotros mismos o sobre el mundo que nos rodea con precisión y objetividad. No posee otra finalidad intrínseca que hacer crecer el conocimiento, que hacer más robusto y frondoso el árbol de la ciencia

-Investigación aplicada: el concepto es idéntico al de investigación básica, se origina y ensambla en ella. Se diferencia de ella en la finalidad: la investigación aplicada está dirigida a algún fin práctico, muy frecuentemente lucrativo. En el contexto de la presente conferencia, centrada en investigaciones biomédicas, podríamos decir que la investigación es básica cuando no tiene conexión directa con una enfermedad concreta, y es aplicada en el caso contrario.

Idealmente, la investigación básica culmina con un descubrimiento y la investigación aplicada culmina con un invento. El descubrimiento hace referencia a algo existente; el invento hace referencia a algo nuevo. Se descubre algo que ya existe, pero que esta oculto; se inventa algo que no existe, p.e., una máquina nueva. 
Tabla 1. Agrupación de los países latinoamericanos en función de sus rentas per capita.

\begin{tabular}{l|l|l|l}
\hline $\mathbf{\$ 5 0 0 0}$ & $\geq 2000$ & $\geq 1000$ & $<1000$ \\
\hline Argentina & Colombia & Guatemala & Bolivia \\
\hline Brasil & Panamá & Paraguay & Honduras \\
\hline Chile & Venezuela & Ecuador & Nicaragua \\
\hline México & R. Dominicana & El Salvador & \\
\hline Uruguay & Perú & Cuba & \\
\hline & Panamá & & \\
\hline & Costa Rica & & \\
\hline
\end{tabular}

gación en Ciencias Fisiológicas. Como científicos, las contestaciones a priori son positivas, porque así lo sentimos, pero como científicos también tenemos que buscar argumentos claros e incontrovertibles que hagan ver esa necesidad a los gobernantes. Estamos hablando de países con rentas per capita muy bajas (Tabla 1) y por tanto con muchas deficiencias. La duda razonable es saber si hacer investigación es una urgencia, o si por el contrario es un lujo prescindible o que se pueda posponer. Para ponerlo en términos operativos la pregunta sería: imaginémonos que los gobernantes de uno de estos países nos preguntaran las razones por las que la investigación es necesaria.

Yendo de lo general a lo particular, comenzaría por aconsejar a los gobernantes del país hipotético que una política general presidida por un liberalismo económico a ultranza no es la aconsejable para los países en desarrollo. El desarrollo de un país basado en un liberalismo económico no hace sino ahondar en las diferencias sociales existentes de partida. Las inversiones estatales se centran en financiar grandes obras, con lo que los beneficios derivados del propio desarrollo revierten de forma insolidaria en el propio capital, en las grandes empresas nacionales o extranjeras, y los ciudadanos de los estratos sociales bajos no se benefician en absoluto del espejismo de desarrollo que esta aconteciendo. Las bolsas de pobreza persisten, los índices de analfabetismo no mejoran, las carencias en asistencia sanitaria se mantienen o se acrecientan, el índice de desempleo no mejora a largo plazo. Y ello a pesar de que existan grandes autopistas que crucen los países; en torno a ellas suele concentrarse la pobreza y el hacinamiento. Por el contrario, un modelo de desarrollo de corte social, en el que las inversiones estatales estén dirigidas a quitar el hambre, a promover la sanidad, a facilitar el acceso a la cultura y al conocimiento, es solidario ya que hace partícipes a todos los ciudadanos, y más a aquellos que más lo necesitan, de las propias rique- zas del país. La inversión en creación de conocimiento desde la alfabetización hasta la enseñanza superior y la investigación científica y tecnológica constituyen objetivos prioritarios de las mismas. Así pues, es evidente que tenemos que invertir en desterrar el hambre y la enfermedad, o lo que es lo mismo invertir en sanidad y en salubridad, pero también hay que invertir en escuelas, en Universidades y en Laboratorios. En este modelo la investigación, como inversión en conocimiento y cultura que es, tiene cabida desde un principio, desde el primer euro peseta de inversión. En el modelo liberal, cuando menos, la inversión en cultura y conocimiento, en investigación se pospone, ya que se trata de una inversión que no da beneficios inmediatos.

Dejando a un lado estos aspectos generales, porque soy un profano en teorías políticas, en mi intento de persuadir a los gobernantes de ese país hipotético bajaría a la arena de lo concreto, para proporcionar razones específicas que aconsejan la promoción de la investigación en los países en desarrollo (cuadro 4).

Cuadro 4. Razones para promover la investigación en los países de Latinoamérica

- Hay una inversión previa en la creación de científicos (Latinoamérica y Europa exportadores de científicos)

- El quehacer de los científicos genera desarrollo y riqueza, es rentable.

- Permite el aprovechamiento directo y gratuito de recursos internacionales (OMS)

- Posibilita acceder al bien gratuito e internacional que es el propio conocimento científico como motor de desarrollo.

- La producción científica de un país es una actividad creativa como la música o el arte y por tanto es irrenunciable porque forma parte de la identidad cultural de dicho país.

Muchas de estas razones se entenderán mejor desde la realidad concreta que presenta El Perú, un país que se encuentra, por su renta per capita, en el tramo medio del ranking dentro de los países latinoamericanos. Existe una Red Mundial de Científicos Peruanos que ahora mismo cuenta con más de 2000 científicos distribuidos por todo el mundo. La existencia de esta misma Red demuestra que en Perú hay Universidades con capacidad para formar científicos, excepto tal vez en sus etapas últimas. No sería necesario a priori crear Universidades porque las que tienen ya generan más universitarios-precientíficos de los que puede absorber el sistema peruano de Ciencia. Por tanto es necesario incidir en ese sistema de cien- 
cia peruano para que absorba el mayor número de científicos posible dentro de los que produce. Y ¿por qué?, porque absorberlos es rentable, ayuda al desarrollo del propio país. Pensemos que en esos 2000 peruanos que conforman esa Red Mundial de Científicos Peruanos, el estado peruano ha invertido dinero en ellos al menos durante 25 años, desde su nacimiento hasta acabar sus estudios universitarios. $\mathrm{Y}$ ahora cuando están en condiciones de producir, han tenido que quedarse fuera; si fuera del Perú, principalmente en los EE.UU. se les acepta es porque son capaces de generar riqueza ${ }^{3}$, y generan riqueza a través de la generación de conocimiento haciendo investigación. Es obvio que el Perú no tiene recursos para recuperar a esos 2000 científicos en diáspora por todo el mundo, ni para pagar sus salarios ni para dotarles y gestionarles sus laboratorios. Sin embargo, sí que podría implementarse una política progresiva y progresista, realista, seria y consensuada a medio y largo plazo que permitiera que ese despilfarro de recursos que el Perú esta haciendo (criar peruanos durante 25 años para que en 2-4 años más alcancen el óptimo de su capacidad productiva y luego dejarlos irse) se redujera hasta llegar a crear un balance entre la formación en sus Universidades de titulados superiores y la asimilación por el propio sistema peruano de esos titulados. No vayan a pensar que este despilfarro es un mal exclusivo de Perú, este es un mal de toda Latinoamérica y aún de toda Europa con relación a los EE.UU. En suma, las primeras razones específicas que yo daría a los gobernantes de nuestro país hipotético de porqué hay que hacer ciencia, de porqué hay que investigar, sería porque poseemos los científicos casi totalmente formados, porque hemos hecho una inversión para criarlos y crearlos y porque los científicos son rentables como demuestra el hecho de que nos los compren, precisamente, por su rentabilidad.

Yo continuaría diciéndoles a los gobernantes de nuestro país hipotético que hay que crear laboratorios por otras razones. Además de la capacidad genérica de la ciencia para promover desarrollo, la ciencia es capaz de traer ingresos directos. Ya he mencionado como la WHO tiene programas con presupuestos específicos para países en desarrollo que no se llegan a implementar en esos países por no poseer laboratorios con un mínimo de garantías de GLP. Pero es que además, sino se posibilita la investigación, sino se crean laboratorios, no se puede acceder a la ciencia, que es un bien generador de riqueza y bienestar y que, especialmente en sus aspectos básicos, es patrimonio de la humanidad. $\mathrm{Si}$ un país no tiene un programa de desarrollo científico adecuado, no puede participar de forma gratuita del conocimiento que se ha creado por los países desarrollados a costa de grandes inversiones.

Finalmente, habría que invertir en investigación porque esta inversión es una inversión en cultura. La investigación, de un lado, como actividad de creación intelectual que es, y de otro, por su origen tribal entre la magia y el arte, constituye junto con el arte y la música la expresión más sublime de los seres humanos, lo que define al homo sapiens. La producción científica de un país, junto con su producción artística, constituye los elementos definitorios de la identidad cultural de ese país. Esta idea de la investigación como elemento definitorio de la identidad nacional es desarrollada por Cajal en varios de sus escritos ${ }^{4}$.

Supongamos que con estos argumentos ya hemos convencido a los gobernantes de nuestro país hipotético que hay que hacer investigación. Hecha esta asunción la pregunta que seguro nos formularían sería, ¿y en que investigamos?. Yo les diría que lo que hay que hacer es sentar las bases para hacer una investigación buena (cuadro 6), que es la única inves-

Cuadro 6. Bases para una buena investigación. Líneas de política científica consensuada a medio y largo plazo

- Hay una inversión previa en la creación de científicos (Latinoamérica y Europa exportadores de científicos)

- El quehacer de los científicos genera desarrollo y riqueza, es rentable.

- Permite el aprovechamiento directo y gratuito de recursos internacionales (OMS)

- Posibilita acceder al bien gratuito e internacional que es el propio conocimento científico como motor de desarrollo. - La producción científica de un país es una actividad creativa como la música o el arte y por tanto es irrenunciable porque forma parte de la identidad cultural de dicho país.

3. Aunque el ultraliberalismo económico que impregna la sociedad norteamericana hace absolutamente sólida la afirmación, no es menos cierto que se acepta a los investigadores de todo el mundo para ejercer sus actividades en los EE.UU. porque los americanos prefieren otras profesiones más lucrativas y menos demandantes, y por tanto más respetadas, tales como abogados, la economía (business) o medicina.

4. Así en Recuerdos de mi vida: Historia de mi labor científica, Cajal, no sin cierto nivel de exaltación, escribe: “...Las dos grandes pasiones del hombre de ciencia son el orgullo y el patriotismo. Trabajan, sin duda, por amor a la verdad, pero laboran aún más en pro de su prestigio personal o de la soberanía intelectual de su país".... 
tigación generadora de cultura y de riqueza, y que hay que tener cuidado de no hacer una mala investigación que lo único que hace es generar giga bits de ruido.

La política de nuestros gobernantes debería empezar por hacer posible la recuperación progresiva de los científicos formados fuera para recuperar parte de la inversión ya hecha. Se crearían programas de recuperación-formación- dotación de recursos. Esto es, se establecería una política científica que no existe en la generalidad de los países en desarrollo, donde la ciencia que se hace se hace por voluntarismo, sin unas líneas maestras definidas, sin ayudas institucionales ni reconocimientos. En la historia reciente de España, pues nos separan menos de treinta años de esta situación, nos ha tocado vivir en nuestros maestros, y aún en carne propia esta carencia, tan fecunda en crear desánimo, en generar sensación de minusvalía e incapacidad intelectual. En el establecimiento de esa política científica habría que tener en cuenta las GLP internacionales que he mencionado anteriormente.

Bueno, ya los hemos convencido, hay que hacer investigación de la buena, pero, ¿y en qué áreas?. En este apartado yo diría que habrían de ser tajantes nuestros gobernantes: habría de hacerse investigación en aquellas parcelas de la biomedicina que tienen una incidencia particular en el propio país. ¿Dónde se van a investigar las enfermedades tropicales?. En este mundo insolidario en que vivimos, tenemos que pensar que si la malaria no afecta a los países del Primer Mundo, no va a haber, en los países del Primer Mundo, una investigación decidida para resolver el problema de la malaria. Lo mismo ocurre con la fisiología y medicina de grandes alturas. La medicina de grandes alturas afecta de forma específica a unas pocas regiones del globo, pero la mayor parte de la población que vive a grandes alturas lo hace en la franja andina: más del $80 \%$ de los 16-20 millones de personas que viven por encima de $2500 \mathrm{~m}$ en todo el mundo lo hace en la falda occidental de la cordillera andina. Los problemas específicos de la fisiología y la medicina de grandes alturas sería otra parcela prioritaria en algunos de los países de Latinoamérica. Aún siendo conscientes de que los países pequeños, no pueden abarcar todas las ramas del saber en sus programas de investigación, sí que habría que hacer esfuerzos para abrir el abanico de forma progresiva para, partiendo de esas áreas prioritarias, incidir en un número creciente de parcelas del saber.

La pregunta siguiente sería ¿hacemos investigación básica o aplicada?. De nuevo aquí yo creo que sólo hay un tipo de investigación, que la investiga- ción básica y la aplicada son la misma cosa en distintos estadios de crecimiento (ver nota al pie de página número 2, pàg 45; Figura 1). Es miópico pensar que sin investigación básica se pueda crear investigación aplicada. Es imposible diseñar una vacuna o un tratamiento contra la malaria si no sabemos cómo es y cómo funciona el plasmodio.

Un último aspecto que vigilaría como gobernante sería el proceso de gestión de la investigación para evitar su burocratización. La burocracia en su significado peyorativo se refiere a una administración ineficiente a causa del papeleo, la rigidez y las formalidades superfluas. La burocratización es tan nociva que podríamos compararla a un tejido que crece de forma independiente y autónoma hasta convertirse en un tumor, y que en el proceso pierde la función para la que estaba diseñado y adquiere una función autónoma y propia, que no es otra que asegurar su propia supervivencia, entorpeciendo la función del órgano principal.

Figura 1. Comparación de la investigación básica y aplicada con el desarrollo humano

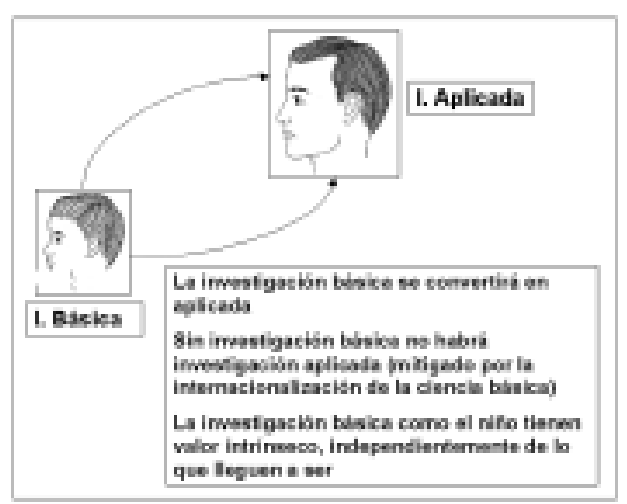

Pues bien, cuando la administración dedicada a la investigación se burocratiza, los burócratas olvidan su finalidad y, en una tarea autocomplaciente y narcisista, generan formularios, regulaciones, e inspecciones, estorbos todos, que exigen gran esfuerzo al investigador y que le obligan a repartir sus energías entre lo que debe ser su tarea, que es crear ciencia y la tarea estéril de satisfacer el ego narcisista del burócrata. Así el investigador asiste impotente al mandato de llenar formularios que no sabe como rellenar o que cuestan gran tiempo y esfuerzo rellenarlos, leyendo regulaciones crípticas hechas para investigadores por quien no sabe lo que es la investigación, etc. Finalmente, no hay que menos- 
preciar otra propiedad del burócrata que es su poder: el burócrata de turno es capaz de obstaculizar, y con ello se siente realizado, un proceso millonario porque el formulario rellenado no corresponde al de su departamento, sino al del departamento contiguo. El tratamiento que a este punto he dado es tan vehemente, porque en nuestra España, aún con la juventud de su sistema de ciencia regulada, ha habido, especialmente en los últimos cuatro o seis años, un crecimiento desaforado de la burocracia: un intervencionismo asfixiante que nos fuerza a un gasto innecesario de energías, y un espíritu fiscalizador que nos sitúa siempre a los investigadores bajo el punto de mira de la sospecha ${ }^{5}$.

Finalmente, y con ello llego al título de mi charla: ¿qué hay de la investigación en Ciencias Fisiológicas?. Creo que todos estaríamos de acuerdo en que la Fisiología, junto con la Anatomía y la Histología (así lo reclamaron los colegas chilenos y mejicanos que eran anatómicos e histólogos), constituyen la base de la Medicina. La Fisiología es una ciencia básica y, por tanto, hacer investigación en Fisiología, es hacer investigación básica. Es imposible investigar y entender la fisiopatología y la Medicina sin conocer la Fisiología de los procesos normales. Huelgan más explicaciones para defender la investigación fisiológica. Pero, para finalizar mi conferencia, sí que quiero decir unas pocas cosas sobre los niveles de estudio de las Ciencias Fisiológicas y sobre la investigación en esos niveles. Trato de referirme al nivel de animal entero, de órganos y aparatos, de células, de moléculas. Hay que cubrir todo el abanico. Sin embargo hay que ser conscientes de que según los niveles, o según enfoques dentro de un mismo nivel, las investigaciones son más caras o más baratas. En general, la investigación a nivel molecular es más cara que a nivel organismal, y en cualquier caso, el enfoque bioquímico es más caro de sostener que el biofísico. Como estrategia allí donde los recursos son más limitados abogaría por una investigación de corte más biofísico, ya que también con técnicas biofísicas se puede hacer biología molecular.

Finalmente, quiero retomar una idea que daba al principio, y que se refiere al carácter analítico de la investigación actual. Hacen gran falta investigaciones integradoras que vayan ensamblando en el animal intacto el sinnúmero de piezas descritas. En este sentido, hemos de saber que las grandes compañías farmacéuticas tienen dificultades para encontrar investigadores capaces de manejar un animal intacto para obtener información integrada que el desarrollo de los medicamentos exige. Tal vez una orientación de la investigación en esta dirección, i.e., al estudio de las funciones integradas, permitirían ofertar nuestros laboratorios como bancos de pruebas en las investigaciones preclínicas necesarias en el desarrollo de nuevos fármacos (ver Diagrama 1). La investigación fisiológica y la investigación aplicada se dan la mano en el estudio de las acciones farmacológicas y de los mecanismos de acción de cualquier droga. En cualquier caso, sólo se podrán implementar estos estudios si existen unas directrices claras y una evaluación correcta dentro de nuestros países de las GLP emanadas de las organizaciones supranacionales. Gracias.

\section{BIBLIOGRAFÍA (otras fuentes consultadas)}

1. Contreras Nuño, J.G. El valor intrínseco de la investigación básica. Avance y Perspectiva 22: 122-124, 2003.

5. Hay una anécdota real ocurrida en una Universidad Española que ejemplariza este punto y que se la conoce con el nombre del caso del "céntimo de euro". El profesor X recibe una factura por valor de algunos miles de euros de los que el 16\% era el IVA y tras visarla y firmar que estaba de acuerdo la remite al negociado correspondiente para que se le pague al proveedor. A los pocos días recibe la factura devuelta desde el negociado en cuestión porque el IVA estaba mal calculado. La cantidad precisa que en concepto de IVA figuraba en la factura era de 526,47 euros, y el Profesor X al recibir la factura devuelta recalcula el IVA correspondiente al valor del equipo suministrado y le sale una cantidad de 526,475012 euros. Ante esta constatación llama al negociado en cuestión y pide explicaciones al administrativo de turno, quien le informa que en su ordenador el IVA que sale es de 526,48 euros. El Profesor X le explica que mientras que su ordenador ha redondeado por exceso, el proveedor ha redondeado por defecto y que la diferencia en cuestión es medio céntimo de euro. El administrativo insiste que de acuerdo con su programa informático el IVA es de 526,48 euros y que esa es la cantidad que debe figurar en la factura porque de otra forma no le casan luego los estadillos, y que lo que debe hacer es pedir al proveedor que le rehaga la factura poniéndole el IVA correcto, que si no lo hace así que no paga la factura. El Profesor X indignado llama por teléfono al proveedor y tras comentarios jocosos y felicitarse la Navidad, el proveedor queda en enviarle la nueva factura con el IVA correcto en breve. Dan las vacaciones de Navidad y el Profesor en cuestión ve la nueva factura a su regreso de vacaciones y tras visarla la envía de nuevo al negociado de pagos. En la segunda quincena de enero recibe devuelta la factura porque tiene fecha del año anterior y estamos en un ejercicio económico nuevo. Nueva llamada telefónica del Profesor X al proveedor: esta vez para explicarle que ha de hacer una factura nueva con la fecha del año en curso. Obviamente la conversación no es jocosa ni tiene espíritu navideño. Finalmente, tras un gasto importante en sobres, correo, llamadas telefónicas, tiempo del administrativo de la Universidad, tiempo del Profesor X, tiempo del proveedor y su personal de oficina y varios meses de retraso, la factura se paga y se resuelve el problema del medio céntimo de euro. El caso ejemplariza el daño que un exceso de celo puede ocasionar. 
2. Deis, RP. La investigación básica en América Latina. Acta Andina 9: 65-67, 2001. Dirección electrónica:

http://sisbib.unmsm.edu.pe/BVRevistas/Acta_Andina/vol9_n1y2

3. Embajada de Noruega en España. Educación e Investigación: Investigaciones básicas a largo plazo. 2003. Dirección electrónica: http://noruega.es/education/research/basic/basic.htm

4. Jiménez de Castro, G. Orientándonos por los quasares: el valor de la Investigación Básica. Ciencia Hoy :agosto 2000. Obtenido a través Internet:

http://www.craam.mackenzie.br/ guigue/papers/Quasars/Quasares
5. Tiemblo, A. Investigación básica y aplicada. 1999. Obtenido de internet: http://csic.es/asociaciones/api/libro/investigacion.htm

6. Acuña, MD. La Investigación científica y el desarrollo de los países 2002. Obtenido de Internet:

http://luventicus.org/articulos/02R011/

7. National Institute of General Medical Sciences. News \& Events: Why do basic research?. 1997. Obtenido en Internet: http://www.nigms/nih.gov/news/science_ed/whydo/html

8. Los datos relativos a los PIB y Rentas per Capita fueron obtenidos de Internet en múltiple páginas.

\section{Sesión de trabajo \\ Prof. E. Suárex, Prof. J. Machuca, Orof. F. González, Prof. L. Maldonado, y Profa. V. Hortale}

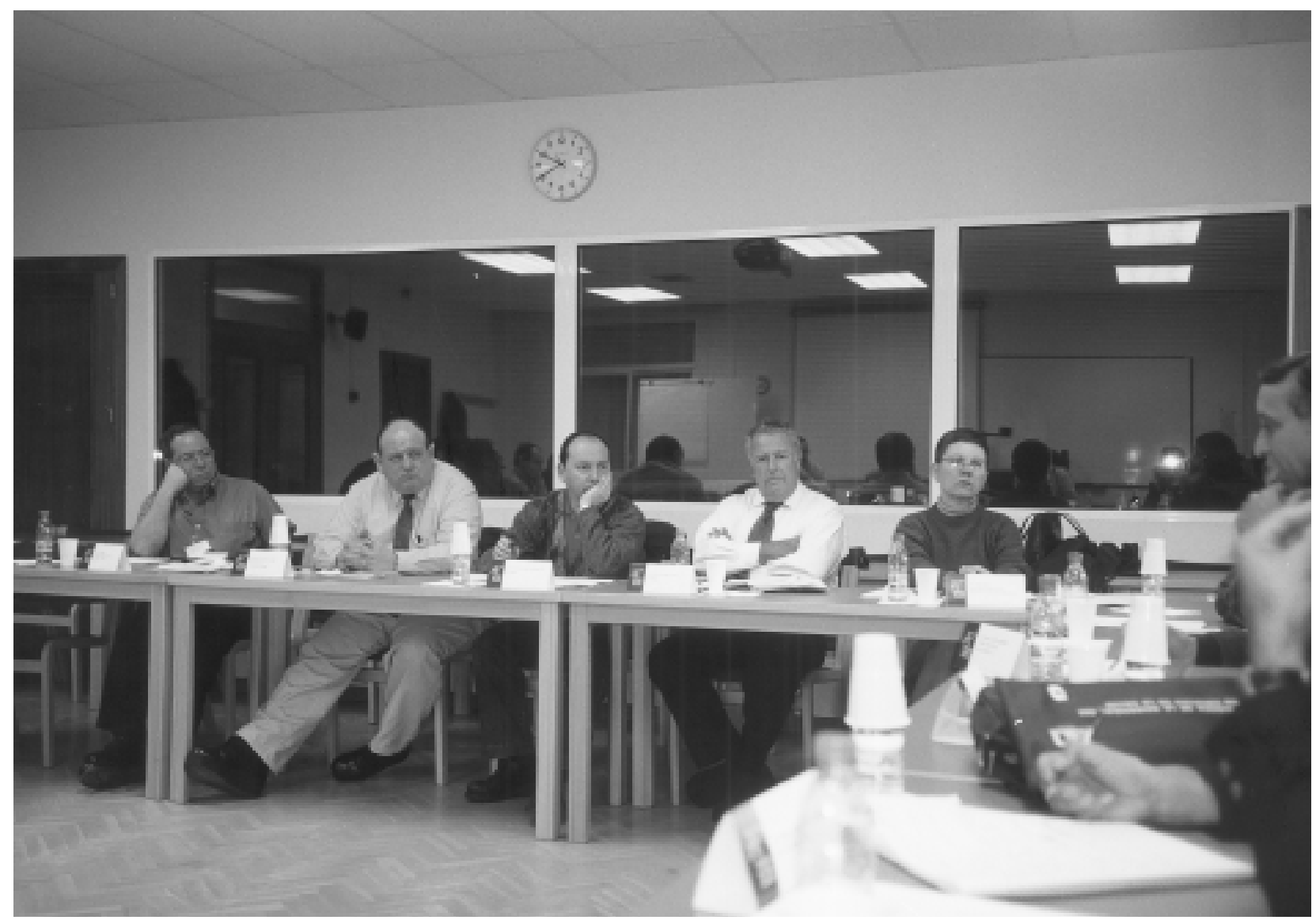

